\title{
Vor neuen Herausforderungen
}

\begin{abstract}
Wohl kein anderes Politikfeld ist in den letzten Jahren mit so grundlegend veränderten Rahmenbedingungen konfrontiert worden wie die Energiepolitik. Der auf Druck der Europäischen Union (EU) zustande gekommene Liberalisierungsprozess des Strom- und Gassektors ist jedoch mit gravierenden Problemen verbunden. Der Handlungsbedarf für die europäische Energie- und Umweltpolitik ist erheblich.
\end{abstract}

$\mathrm{D}$

Von Felix Christian Matthes ie Liberalisierungsprozesse sind bisher sehr asymmetrisch verlaufen. Bereits vier Jahre nach Inkrafttreten der Richtlinie über den Strom-Binnenmarkt sind die Folgen so groß, dass die Europäische Kommission eine Beschleunigung und stärkere Regulierung der Marktöffnung angekündigt hat. Der Problemdruck ergibt sich vor allem aus zwei Entwicklungen.

Erstens hat die sehr ungleiche Öffnung der Strommärkte in den verschiedenen Staaten dazu geführt, dass Energieversorger aus Staaten mit nur geringer Marktöffnung mit den Monopolgewinnen aus noch nicht geöffneten Marktsegmenten im Ausland aggressive Expansionsstrategien finanzieren können. Im Mittelpunkt der Kritik steht hier insbesondere die französische EdF.

Zweitens ist die praktische Ausgestaltung des Kernstücks der Liberalisierung, die öffnung der Übertragungs- und Verteilungsnetze, in einigen Staaten ausgesprochen unbefriedigend gelöst. Vor allem trifft dieser Vorwurf Deutschland, das als einziges EU-Land keine staatliche Regulierung des Netzzugangs etabliert hat, sondern auf frei verhandelten Netzzugang und freiwillige Vereinbarungen mit der Wirtschaft gesetzt hat.

Die energiepolitischen Folgen der Strommarktliberalisierung sind einschneidender Art. Das Strompreisniveau hat sich in fast allen europäischen Staaten und für nahezu alle Kundengruppen signifikant verringert, auch komplementäre umweltpolitische Instrumente wie die in diesem Bereich durchaus ambitionierte Stromsteuer in Deutschland haben diesen Prozess nicht kompensieren können. Die Energieversorgungsunternehmen konzentrieren sich stark auf den Energieverkauf, die in der ersten Hälfte der neunziger Jahre durchaus beachtlichen Aktivitäten im Energiesparsektor sind nahezu vollständig zum Erliegen gekommen. Umfangreiche Kraftwerkskapazitäten werden stillgelegt, wobei vor allem die besonders umwelt- freundliche Kraft-Wärme-Kopplung stark betroffen ist. Gleichzeitig ist die Investitionstätigkeit signifikant zurückgegangen, Lebensdauerverlängerungen für Kraftwerke werden in umfangreichem Maße folgen. Dies kann langfristig - wie die aktuellen Entwicklungen in Neuseeland und Kalifornien andeuten - zu erheblichen Problemen in der Versorgungssicherheit führen.

\section{- Umweltpolitik unter Zugzwang}

Diese energiewirtschaftlichen Trends haben wichtige umweltpolitische Konsequenzen, bildet doch die Stromwirtschaft einen herausragenden Verursacherbereich für ökologische Probleme. Etwa ein Drittel der Emissionen des wichtigsten Treibhausgases Kohlendioxid werden in der EU durch die Stromversorgung verursacht, die Menge des bisher produzierten hochradioaktiven Abfalls aus Kernkraftwerken beläuft sich mittlerweile auf mehr als 50.000 Tonnen Schwermetall. Gleichzeitig ist die Europäische Union selbst von den - im Vergleich zur Größe des Klimaproblems - geringen Emissionsminderungszusagen des Kioto-Protokolls (minus 8 Prozent im Mittel der Jahre 2008 bis 2012 gegenüber 1990) noch weit entfernt.

Folgerichtig ergeben sich aus der wettbewerbspolitisch induzierten Strommarktliberalisierung große energie- und umweltpolitische Herausforderungen. Da der EG-Vertrag kein energiepolitisches Kapitel enthält, wachsen der EU nur geringe energiepolitische Kompetenzen $\mathrm{zu}$, während paradoxerweise die energiepolitischen Konsequenzen der EU-Wettbewerbspolitik gravierend sind. Nationale energie- und umweltpolitische Maßnahmen kollidieren regelmäßig mit dem zunehmend strengeren, gleichzeitig aber keineswegs widerspruchsfreien und teilweise intransparenten Wettbewerbsrahmen der EU. Die resultierenden Phasen erheblicher europarechtlicher Unsicherheiten erschweren den nationalen Politikformulierungsprozess oft erheblich.
Der sehr erfolgreich in andere Politikbereiche integrierten Wettbewerbspolitik steht eine europäische Umweltpolitik gegenüber, deren horizontale Integration in vielen Bereichen noch aussteht. Diese Integrationsasymmetrie führt dazu, dass eine ganze Reihe - oft auch sehr innovativer - umweltpolitischer Vorhaben der Mitgliedsstaaten wegen ihres fehlenden EU-Rahmens vor erheblichen wettbewerbsrechtlichen Problemen steht; vergleichsweise ,unauffälligen“ Regelwerken wie dem Gemeinschaftsrahmen für Umweltbeihilfen kommt hierbei eine herausragende Rolle zu (vgl. auch die Nachricht auf S. 2).

Diesen politisch teilweise höchst brisanten Entwicklungen kann nur entgegengewirkt werden, wenn die drängenden energie- und umweltpolitischen Herausforderungen auf EU-Ebene mit gleicher Intensität verankert werden können, wie heute bereits der Binnenmarkt. Im Vordergrund sollten kurz- und mittelfristig vor allem stehen:

- die Schaffung von Rahmenrichtlinien für erneuerbare Energiequellen, die Kraft-WärmeKopplung sowie die Erhöhung der Energieeffizienz (vgl. auch die Nachricht auf S. 2);

- die Schaffung von Regelungen zur Transparenzerhöhung und wettbewerbspolitischen Gleichbehandlung für die verschiedenen energiewirtschaftlichen Subventionsbereiche (vor allem hinsichtlich der Kernenergie);

- die Schaffung EU-weiter umweltpolitischer Instrumentarien, etwa einen Emissionshandel für Treibhausgase.

Wissenschaft und Politik sind heute oft noch national fixiert. Den energie- und umweltpolitischen Herausforderungen werden die in den nächsten Jahren nur dann noch genïgen können, wenn sie sich selbst der Herausforderung Europa stellen (1).

\section{Anmerkung}

(1) Eine ausführliche Darstellung findet sich in Felix Chr. Matthes, Christof Timpe: Sustainability and the Future of European Electricity Policy. Policy paper für die Heinrich-BöllStiffung: Documentations, Papers \& Reports No. 4. Bezug über www.boell.de.

\section{Der Autor}

Dr. Felix Christian Matthes ist wissenschaftlicher Mitarbeiter des Öko-Instituts.

Kontakt: Öko-Institut, Novalisstr. 10,

D-10115 Berlin, Tel. 030/ 280486-80, Fax -88,

E-mail: matthes@oeko.de 
(c) 20I0 Authors; licensee IÖW and oekom verlag. This is an article distributed under the terms of the Creative Commons Attribution Non-Commercial No Derivates License (http://creativecommons.org/licenses/by-nc-nd/3.o/), which permits unrestricted use, distribution, and reproduction in any medium, provided the original work is properly cited. 